\title{
Adaptive LQ Cascade Control of a Tubular Chemical Reactor
}

\author{
Dostal Petr, Vladimír Bobal and Jiri Vojtesek \\ Tomas Bata University in Zlin, Faculty of Applied Informatics, Nad Stranemi 4511, 76005 Zlin, Czech Republic
}

\begin{abstract}
The paper deals with adaptive LQ cascade control design of a tubular chemical reactor with an exothermic consecutive reaction. The control is performed in primary and secondary control-loops where the primary controlled output of the reactor is the concentration of a main reaction product and the secondary output is the mean temperature of the reactant. A common control input is the coolant flow rate. The controller in the primary control-loop is a nonlinear P-controller with the gain calculated using simulated or measured steady-state characteristics of the reactor. The controller in the secondary control-loop is a LQ adaptive controller. The proposed method is verified by control simulations.
\end{abstract}

\section{Introduction}

Tubular chemical reactors (TCRs) are units frequently used in chemical industry, biotechnologies and some others. From the system theory point of view, TCRs belong to the class of nonlinear distributed parameter systems. Their mathematical models are described by sets of nonlinear partial differential equations (PDEs). The methods of modelling and simulation of such processes are described e.g. in [1] or [2]. Detailed analysis of the specific TCR is carried out for example in [3].

Tubular chemical reactors belong to hardly controllable processes. Difficulties associated with their control are given not only by their nonlinearity but also by the fact that concentrations of reactants cannot mostly be measured continuously. Here, the cascade control takes place as a suitable and effective control method.

The cascade control belongs to more complex control structures. It may be applied in such cases where more output variables can be measured and where only one input variable is available to the control. Principles of the cascade control are described e.g. in [4], [5] and [6].

In this paper, the TCR control strategy is based on the fact that concentrations of components of reactions taking place in the reactor depend on the reactant temperature. Moreover, the procedure assumes that the reactant temperature is measured at more points along the reactor from which is subsequently calculated mean reactant temperature. Then, in the cascade control-loop, the concentration of a main product of the reaction is considered as the primary controlled variable, and, the mean reactant temperature as the secondary controlled variable. The coolant flow rate represents a common control input. The primary controller determining the set point for the secondary (inner) control-loop is derived as a nonlinear proportional controller using the steady-state characteristics of the reactor. Since the controlled process is nonlinear, a continuous-time adaptive controller is used as the secondary controller. The procedure for the adaptive control design in the inner control-loop is based on approximation of a nonlinear model of the TCR by a continuous-time external linear model (CT ELM) with recursively estimated parameters. In the process of the parameter estimation, a corresponding delta model is used, see, e.g. [7], [8] and [9]. The resulting CT controller is derived on the basis of the LQ control theory, see, e.g. (10) and the polynomial method, see, e.g. [11] or [12]. The control is tested by simulations on the nonlinear model of the TCR with a consecutive exothermic reaction.

\section{Model of the reactor}

An ideal plug-flow tubular chemical reactor with a simple exothermic consecutive reaction $A \stackrel{k_{1}}{\rightarrow} B \stackrel{k_{2}}{\rightarrow} C$ in the liquid phase and with the countercurrent cooling is considered. Heat losses and heat conduction along the metal walls of tubes are assumed to be negligible, but dynamics of the metal walls of tubes are significant. All densities, heat capacities, and heat transfer coefficients are assumed to be constant. Under above assumptions, the reactor model can be described by five PDEs in the form

$$
\begin{gathered}
\frac{\partial c_{A}}{\partial t}+v_{r} \frac{\partial c_{A}}{\partial z}=-k_{1} c_{A} \\
\frac{\partial c_{B}}{\partial t}+v_{r} \frac{\partial c_{B}}{\partial z}=k_{1} c_{A}-k_{2} c_{B} \\
\frac{\partial T_{r}}{\partial t}+v_{r} \frac{\partial T_{r}}{\partial z}=\frac{Q_{r}}{\left(\rho c_{p}\right)_{r}}-\frac{4 U_{1}}{d_{1}\left(\rho c_{p}\right)_{r}}\left(T_{r}-T_{w}\right)
\end{gathered}
$$




$$
\begin{aligned}
\frac{\partial T_{w}}{\partial t} & =\frac{4}{\left(d_{2}^{2}-d_{1}^{2}\right)\left(\rho c_{p}\right)_{w}}\left[d_{1} U_{1}\left(T_{r}-T_{w}\right)+\right. \\
& +d_{2} U_{2}\left(T_{c}-T_{w}\right] \\
\frac{\partial T_{c}}{\partial t}- & v_{c} \frac{\partial T_{c}}{\partial z}=\frac{4 n_{1} d_{2} U_{2}}{\left(d_{3}^{2}-n_{1} d_{2}^{2}\right)\left(\rho c_{p}\right)_{c}}\left(T_{w}-T_{c}\right)
\end{aligned}
$$

with initial conditions

$$
\begin{aligned}
& c_{A}(z, 0)=c_{A}^{s}(z), c_{B}(z, 0)=c_{B}^{s}(z), T_{r}(z, 0)=T_{r}^{s}(z), \\
& T_{w}(z, 0)=T_{w}^{s}(z), T_{c}(z, 0)=T_{c}^{s}(z)
\end{aligned}
$$

and boundary conditions

$c_{A}(0, t)=c_{A 0}(t)\left(\mathrm{kmol} / \mathrm{m}^{3}\right), c_{B}(0, t)=c_{B 0}(t)\left(\mathrm{kmol} / \mathrm{m}^{3}\right)$,

$T_{r}(0, t)=T_{r 0}(t)(\mathrm{K}), T_{c}(L, t)=T_{c L}(t)(\mathrm{K})$.

Here, $t$ is the time, $z$ is the axial space variable, $c$ stands for concentrations, $T$ for temperatures, $v$ for fluid velocities, $d$ for diameters, $\rho$ for densities, $c_{p}$ for specific heat capacities, $U$ for heat transfer coefficients, $n_{1}$ is the number of tubes and $L$ is the length of tubes. The subscript $(\cdot)_{r}$ stands for the reactant mixture, $(\cdot)_{w}$ for the metal walls of tubes, $(\cdot)_{c}$ for the coolant, and the superscript $(\cdot)^{s}$ for steady-state values.

The reaction rates and heat of reactions are nonlinear functions expressed as

$$
\begin{gathered}
k_{j}=k_{j 0} \exp \left(\frac{-E_{j}}{R T_{r}}\right), \mathrm{j}=1,2 \\
Q_{r}=\left(-\Delta H_{r 1}\right) k_{1} c_{A}+\left(-\Delta H_{r 2}\right) k_{2} c_{B}
\end{gathered}
$$

where $k_{0}$ are pre-exponential factors, $E$ are activation energies, $\left(-\Delta H_{r}\right)$ are reaction enthalpies in the negative consideration and $R$ is the gas constant.

The fluid velocities are calculated via the reactant and coolant flow rates as

$$
v_{r}=\frac{4 q_{r}}{\pi n_{1} d_{1}^{2}}, \quad v_{c}=\frac{4 q_{c}}{\pi\left(d_{3}^{2}-n_{1} d_{2}^{2}\right)}
$$

The parameter values with correspondent units used for simulations are given in Table 1.

From the system engineering point of view, $c_{A}(L, t)=c_{A \text { out }}, \quad c_{B}(L, t)=c_{B \text { out }}, \quad T_{r}(L, t)=T_{r \text { out }} \quad$ and $T_{c}(0, t)=T_{c \text { out }}$ are the output variables and $q_{r}(t), q_{c}(t)$, $c_{A 0}(t), T_{r 0}(t)$ and $T_{c L}(t)$ are the input variables. Among them, for the control purposes, mostly the coolant flow rate can be taken into account as the control variable, whereas other inputs entering into the process can be accepted as disturbances. In this paper, the mean reactant temperature

$$
T_{m}(t)=\frac{1}{n_{p}} \sum_{p=1}^{n_{p}} T_{r}\left(z_{p}, t\right)
$$

is considered as the secondary (inner) controlled output. Here, $z_{p}$ refers to measurement points and $n_{p}$ is their number. The concentration $c_{B}$ represents the primary controlled output.
Table 1. Parameters and input values.

\begin{tabular}{|l|l|}
\hline$L=8 \mathrm{~m}$ & $n_{1}=1200$ \\
\hline$d_{1}=0.02 \mathrm{~m}$ & $d_{2}=0.024 \mathrm{~m}$ \\
\hline \multicolumn{2}{|c|}{$d_{3}=1 \mathrm{~m}$} \\
\hline$\rho_{r}=985 \mathrm{~kg} / \mathrm{m}^{3}$ & $c_{p r}=4.05 \mathrm{~kJ} / \mathrm{kg} \mathrm{K}$ \\
\hline$\rho_{w}=7800 \mathrm{~kg} / \mathrm{m}^{3}$ & $c_{p w}=0.71 \mathrm{~kJ} / \mathrm{kg} \mathrm{K}$ \\
\hline$\rho_{c}=998 \mathrm{~kg} / \mathrm{m}^{3}$ & $c_{p c}=4.18 \mathrm{~kJ} / \mathrm{kg} \mathrm{K}$ \\
\hline$U_{1}=2.8 \mathrm{~kJ} / \mathrm{m}^{2} \mathrm{~s} \mathrm{~K}$ & $U_{2}=2.56 \mathrm{~kJ} / \mathrm{m}^{2} \mathrm{~s} \mathrm{~K}$ \\
\hline$k_{10}=5.61 \cdot 10^{16} 1 / \mathrm{s}$ & $k_{20}=1.128 \cdot 10^{18} 1 / \mathrm{s}$ \\
\hline$E_{1} / R=13477 \mathrm{~K}$ & $E_{2} / R=15290 \mathrm{~K}$ \\
\hline$\left(-\Delta H_{r 1}\right)=5.8 \cdot 10^{4} \mathrm{~kJ} / \mathrm{kmol}$ & $\left(-\Delta H_{r 2}\right)=1.8 \cdot 10^{4} \mathrm{~kJ} / \mathrm{kmol}$ \\
\hline$c_{A 0}^{s}=2.85 \mathrm{kmol} / \mathrm{m}^{3}$ & $c_{B 0}^{s}=0 \mathrm{kmol} / \mathrm{m}^{3}$ \\
\hline$T_{r 0}^{s}=323 \mathrm{~K}$ & $T_{c L}^{s}=293 \mathrm{~K}$ \\
\hline \multicolumn{2}{|c}{$q_{r}^{s}=0.15 \mathrm{~m}^{3} / \mathrm{s}$} \\
\hline
\end{tabular}

For solution of PDEs, the finite differences method is employed. The procedure is based on substitution of the space interval $z \in<0, L>$ by a set of discrete node points $\left\{z_{i}\right\}$ for $i=1, \quad, n$, and, subsequently, by approximation of derivatives with respect to the space variable in each node point by finite differences. The procedure is in detail described in [3].

\section{Control objective}

Basic scheme of the cascade control is in Fig. 1. Here, NPC stands for the nonlinear proportional controller, AC for the adaptive LQ controller.

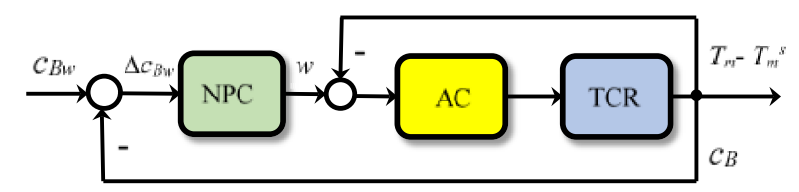

Figure 1. Cascade control scheme.

The control objective is to achieve a concentration of the component $B$ as the primary controlled output near to its maximum. A dependence of the concentration of $B$ on the mean reactant temperature is in Fig. 2.

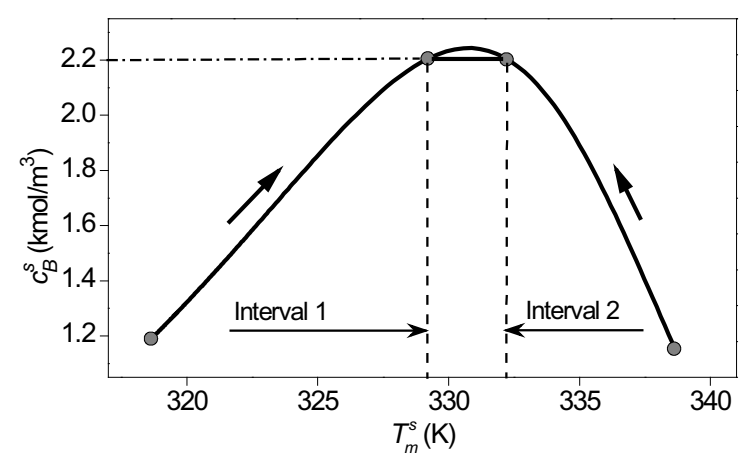

Figure 2. Steady-state characteristics. 
There, the operating interval consists of two parts. In the first interval, the concentration $B$ increases with increasing reactant temperature, in the second interval it again decreases. The endpoints defining both intervals are in Table 2.

It can be seen in Fig. 2 that the maximum value of $c_{B}$ can be slightly higher than $2.2 \mathrm{kmol} / \mathrm{m}^{3}$. However, the maximum desired value of $c_{B}$ will be limited just by 2.2 $\mathrm{kmol} / \mathrm{m}^{3}$.

Table 2. Parameters and input values.

\begin{tabular}{|c|c|c|}
\hline Interval & Temperature & Concentration \\
\hline 1 & $318.7 \leq T_{m} \leq 329.3$ & $1.193 \leq c_{B} \leq 2.2$ \\
\hline 2 & $332.4 \leq T_{m} \leq 338.7$ & $2.2 \geq c_{B} \geq 1.142$ \\
\hline
\end{tabular}

For purposes of later approximations, the mean temperature is transformed as

$$
\xi=\frac{T_{m}-T_{m \min }}{T_{m \max }-T_{m \min }}
$$

where $T_{m \text { min }}=318.7$ and $T_{m \max }=338.7$.

The dependence of $c_{B}$ on $\zeta$ is shown in Fig. 3.

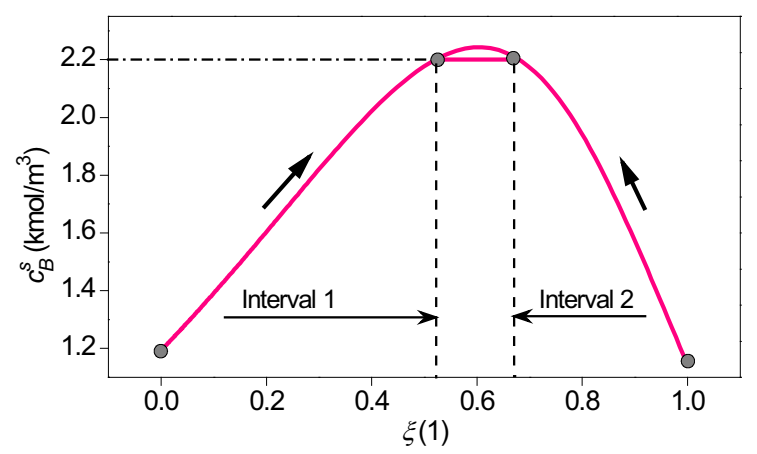

Figure 3. Transformed steady-state characteristics.

\section{The NPC design}

The procedure of the NPC design appears from polynomial approximation of transformed steady-state characteristics shown in Fig. 3. Approximate polynomials in both intervals are stated including their derivatives in Table 3 .

Table 3. Approximate polynomials and their derivatives.

\begin{tabular}{|c|c|}
\hline $\begin{array}{c}\text { Interva } \\
1\end{array}$ & Approximate polynomials \\
\hline \multirow{2}{*}{1} & $c_{B}=1.1954+1.7848 \xi+1.9554 \xi^{2}-3.1818 \xi^{3}$ \\
\cline { 2 - 3 } & $c_{B}^{\prime}=1.7848+3.9108 \xi-9.5454 \xi^{2}$ \\
\hline \multirow{2}{*}{2} & $c_{B}=-6.1051+31.0236 \xi-35.9335 \xi^{2}+12.1564 \xi^{3}$ \\
\cline { 2 - 2 } & $c_{B}^{\prime}=31.023-71.867 \xi+36.4692 \xi^{2}$ \\
\hline
\end{tabular}

Now, a desired value of the mean reactant temperature in the output of the NPC can be computed for each $c_{B}$ as

$$
w=K_{w}\left(T_{m \max }-T_{m \min }\right)\left(\frac{d c_{B}}{d \xi}\right)^{-1} \Delta c_{B w}
$$

where $\Delta c_{B w}=c_{B w}-c_{B}, \quad w=\Delta T_{m w}, \quad$ and $K_{w}$ is a selectable gain coefficient.

\section{CT and Delta external linear model}

For the control purposes, the controlled output and the control input are defined as

$$
u(t)=\Delta q_{c}(t)=q_{c}(t)-q_{c}^{s}, \quad y(t)=\Delta T_{m}(t)=T_{m}(t)-T_{m}^{s}
$$

The CT ELM is proposed in the time domain on the basis of preliminary simulated step responses in the form of the second order differential equation

$$
\ddot{y}(t)+a_{1} \dot{y}(t)+a_{0} y(t)=b_{0} u(t)
$$

and, in the complex domain, as the transfer function

$$
G(s)=\frac{b_{0}}{s^{2}+a_{1} s+a_{0}} .
$$

Establishing the $\delta$ operator

$$
\delta=\frac{d-1}{T_{0}}
$$

where $\delta$ is the forward shift operator and $T_{0}$ is the sampling period, the delta ELM corresponding to (12) takes the form

$$
\delta^{2} y\left(t^{\prime}\right)+a_{1}^{\prime} \delta y\left(t^{\prime}\right)+a_{0}^{\prime} y\left(t^{\prime}\right)=b_{0}^{\prime} u\left(t^{\prime}\right)
$$

where $t^{\prime}$ is the discrete time.

When the sampling period is shortened, the delta operator approaches the derivative operator, and the estimated parameters $a^{\prime}, b^{\prime}$ reach the parameters $a, b$.

\section{Delta model parameter estimation}

Substituting $t^{\prime}=k-2$, equation (15) can be rewriten to the form

$$
\delta^{2} y(k-2)+a_{1}^{\prime} \delta y(k-2)+a_{0}^{\prime} y(k-2)=b_{0}^{\prime} u(k-2)(16)
$$

Establishing the regression vector

$$
\boldsymbol{\Phi}_{\delta}^{T}(k-1)=(-\delta y(k-2) \quad-y(k-2) \quad u(k-2))
$$

where

$$
\delta y(k-2)=\frac{y(k-1)-y(k-2)}{T_{0}}
$$

the vector of delta model parameters

$$
\boldsymbol{\Theta}_{\delta}^{T}(k)=\left(\begin{array}{lll}
a_{1}^{\prime} & a_{0}^{\prime} & b_{0}^{\prime}
\end{array}\right)
$$

is recursively estimated by the least squares method with exponential and directional forgetting from the ARX model, e.g. [12].

$$
\delta^{2} y(k-2)=\boldsymbol{\Theta}_{\delta}^{T}(k) \boldsymbol{\Phi}_{\delta}(k-1)+\varepsilon(k)
$$

where 


$$
\delta^{2} y(k-2)=\frac{y(k)-2 y(k-1)+y(k-2)}{T_{0}^{2}} .
$$

\section{Adaptive LQ controller}

The secondary feedback control loop is depicted in Fig. 4. In the scheme, $w$ is a sequence of step reference signals as outputs from the primary controller, $e$ is the tracking error, $u$ is output of the secondary controller, and $y$ is the controlled output. The transfer function $G(s)$ of the CT ELM is given by (13).

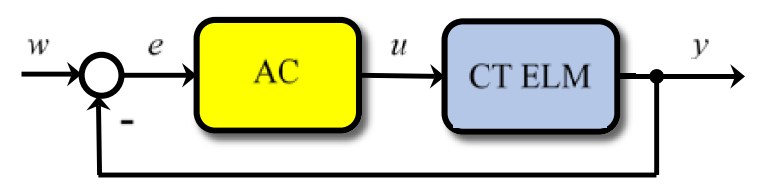

Figure 4. Feedback control loop.

The controller design is based on the polynomial approach and the LQ control theory. Procedure for designing can be briefly described as follows:

The transfer function of the $\mathrm{AC}$ is in the form

$$
Q(s)=\frac{q(s)}{p(s)}
$$

where $q$ and $p$ are coprime polynomials satisfying the condition of properness $\operatorname{deg} q(s) \leq \operatorname{deg} p(s)$. Such control law is sought that minimizes the quadratic cost function

$$
S=\int_{0}^{\infty}\left\{e^{2}(t)+\varphi \dot{u}^{2}(t)\right\} d t
$$

where $\dot{u}(t)$ is the controller output derivative and $\varphi$ is the weighting coefficient.

As known, the problem is solved by controller whose polynomials are given by a solution of the polynomial equation

$$
a(s) p(s)+b(s) q(s)=d(s)
$$

with a stable polynomial $d(s)$ on the right side, and, where $p(s)=s \tilde{p}(s)$ for a step input signal.

Now, the polynomial $d(s)$ takes the form

$$
d(s)=g(s) n(s)
$$

where $g(s)$ is a monic form of the polynomial $h(s)$ given by spectral factorization

$$
[s a(s)]^{*} \varphi[s a(s)]+b^{*}(s) b(s)=h^{*}(s) h(s) .
$$

The second polynomial $n(s)$ ensuring properness of the controller can be chosen as a result of spectral factorization

$$
a^{*}(s) a(s)=n^{*}(s) n(s) .
$$

For $G(s)$ with $a(s)=s^{2}+a_{1} s+a_{0}$, polynomials $h(s), g(s)$ and $n(s)$ take forms

$$
\begin{gathered}
h(s)=h_{3} s^{3}+h_{2} s^{2}+h_{1} s+h_{0} \\
g(s)=s^{3}+g_{2} s^{2}+g_{1} s+g_{0}, g_{j}=h_{j} / h_{3}
\end{gathered}
$$

$$
n(s)=s^{2}+n_{1} s+n_{0}
$$

The polynomial $d(s)$ has the form

$$
d(s)=s^{5}+d_{4} s^{4}+d_{3} s^{3}+d_{2} s^{2}+d_{1} s+d_{0}
$$

where

$$
\begin{gathered}
d_{0}=g_{0} n_{0}, d_{1}=g_{1} n_{0}+g_{0} n_{1}, d_{2}=g_{2} n_{0}+g_{1} n_{1}+g_{0} \\
d_{3}=g_{3} n_{0}+g_{2} n_{1}+g_{1}, d_{4}=g_{3} n_{1}+g_{2}
\end{gathered}
$$

Then, the resulting strictly proper controller has the transfer function

$$
Q(s)=\frac{q_{2} s^{2}+q_{1} s+q_{0}}{s\left(s^{2}+p_{1} s+p_{0}\right)}
$$

with parameters computed as

$$
\left(\begin{array}{ccccc}
1 & 0 & 0 & 0 & 0 \\
a_{1} & 1 & 0 & 0 & 0 \\
a_{0} & a_{1} & b_{0} & 0 & 0 \\
0 & a_{0} & 0 & b_{0} & 0 \\
0 & 0 & 0 & 0 & b_{0}
\end{array}\right) \times\left(\begin{array}{c}
p_{1} \\
p_{0} \\
q_{2} \\
q_{1} \\
q_{0}
\end{array}\right)=\left(\begin{array}{c}
d_{4}-a_{1} \\
d_{3}-a_{0} \\
d_{2} \\
d_{1} \\
d_{0}
\end{array}\right)
$$

The above procedure implies that the secondary controller parameters can be adjusted by single selectable parameter $\varphi$.

\section{Simulations}

All simulations were performed on nonlinear model of the TCR (1) - (5) with parameters in Table 1.. The concentration $c_{B}$ is measured in the period $\tau_{s}(\mathrm{~s})$. The aim of simulations is to show an effect of adjustable gain $K_{w}$, an effect of the period $\tau_{s}$ and an effect of adjustable weighting parameter $\varphi$ on some control responses. At the start of simulations, the $\mathrm{P}$ controller with a small gain was used. For the $\delta$-model parameter recursive identification, the sampling period $T_{0}=0.5 \mathrm{~s}$ was chosen. In all cases, the reference signal $w$, the mean temperature $T_{m}$ and the concentration $c_{B}$ responses were simulated.

Starting value of the coolant flow rate was always chosen as $q_{c}^{s}=0.48 \mathrm{~m}^{3} / \mathrm{s}$ and to it corresponding initial values $c_{B}^{s}=1.194 \mathrm{kmol} / \mathrm{m}^{3}$ and $T_{m}^{s}=318.68 \mathrm{~K}$. The desired value of $c_{B}$ has been chosen as $c_{B w}=2.1 \mathrm{kmol} / \mathrm{m}^{3}$.

Effect of the parameter $K_{w}$ on above responses is evident Figs. $5-7$. It can be seen that an increasing $K_{w}$ accelerates all signals in the control loop. However, its value is not unrestricted and its convenient value should be found experimentally.

An effect of the period $\tau_{s}$ can be seen in Figs. $8-10$. Although shortening $\tau_{s}$ leads to faster control responses, its length is not freely selectable but it is determined by possibilities of a measurement.

The last group of simulations shows an effect of selectable weight coefficient $\varphi$ on simulated responses. It can be seen that its influence is little significant. This fact is caused by small steps in the sequence of the reference. 


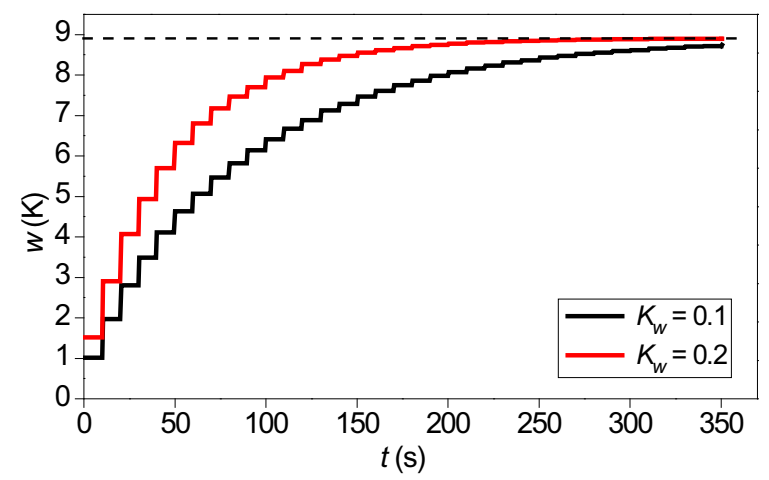

Figure 5. Sequence of step references $\left(\tau_{s}=10, \varphi=100\right)$.

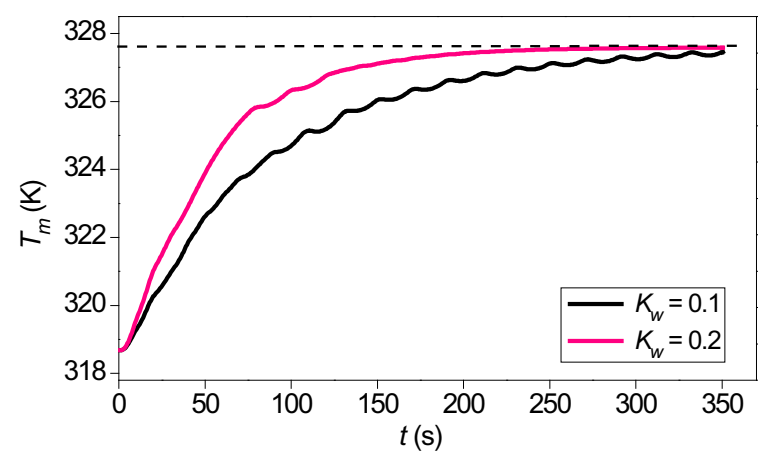

Figure 6. Mean reactant temperature responses $\left(\tau_{s}=10\right.$, $\varphi=100)$.

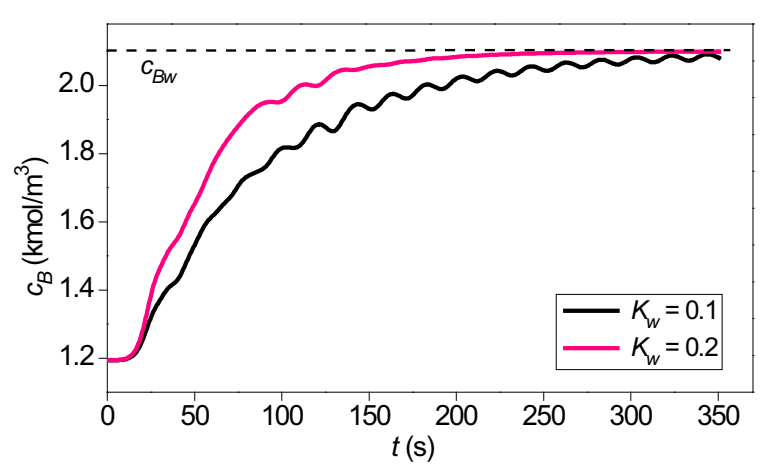

Figure 7. Concentration $c_{B}$ responses $\left(\tau_{s}=10, \varphi=100\right)$.

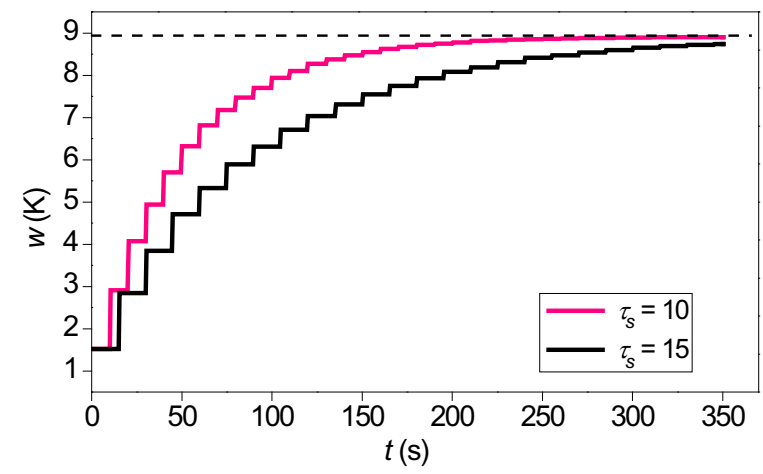

Figure 8. Sequence of step references $\left(K_{w}=0.15, \varphi=100\right)$.

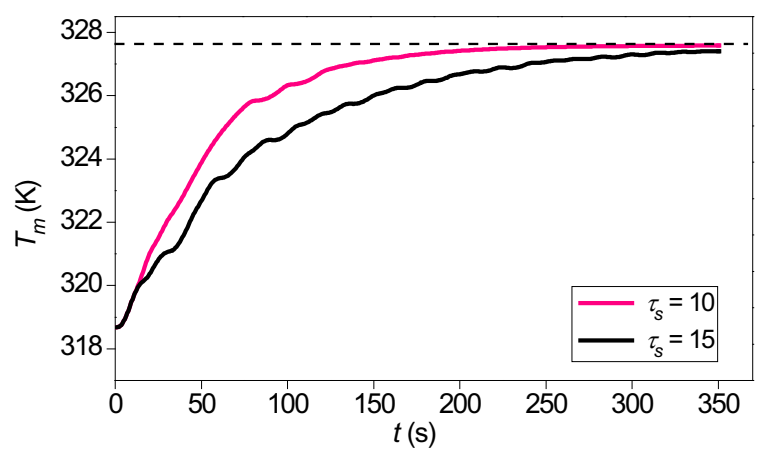

Figure 9. Mean reactant temperature responses $\left(K_{w}=0.15\right.$, $\varphi=100)$.

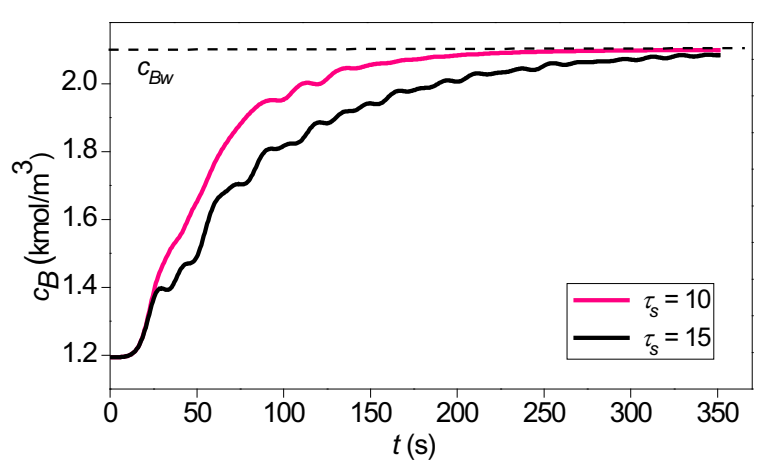

Figure 10. Concentration $c_{B}$ responses $\left(\tau_{s}=10, \varphi=100\right)$.

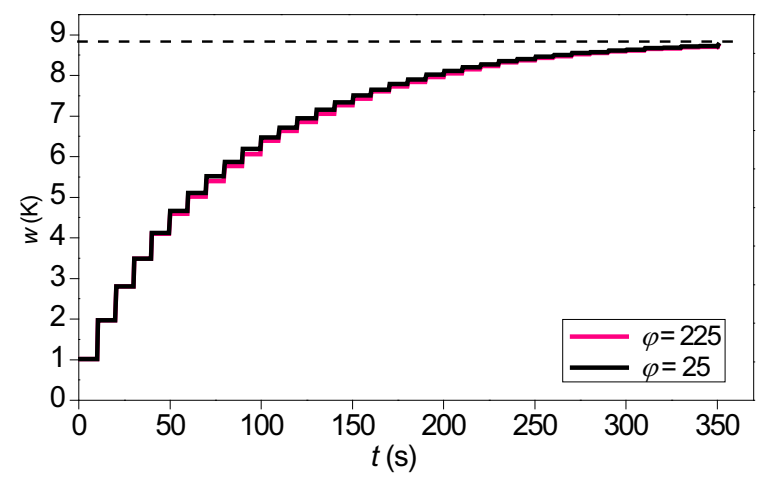

Figure 11. Sequence of step references $\left(K_{w}=0.15, \tau_{s}=10\right)$.

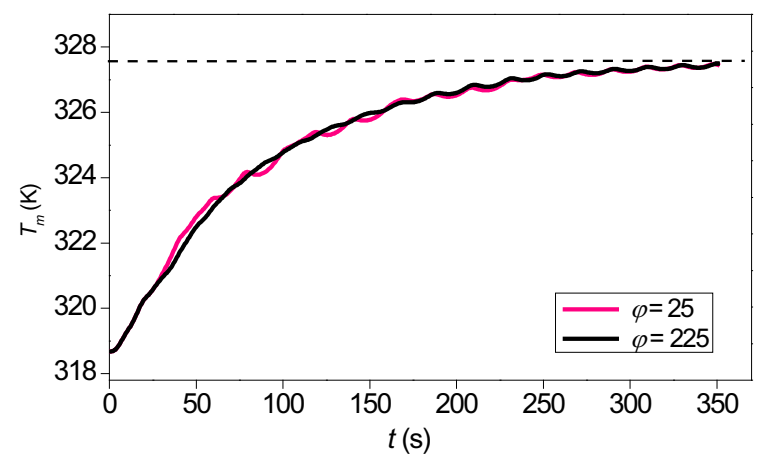

Figure 12. Mean reactant temperature responses $\left(K_{w}=0.1\right.$, $\left.\tau_{s}=10\right)$. 


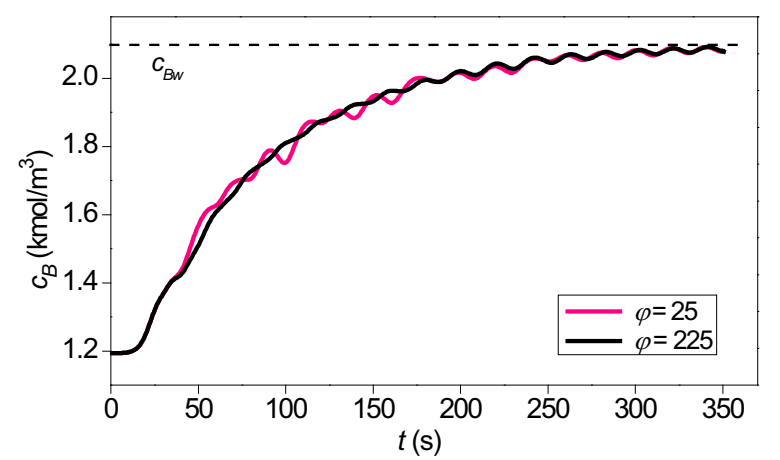

Figure 13. Concentration $c_{B}$ responses $\left(K_{w}=0.1, \tau_{s}=10\right)$.

\section{Conclusions}

The article presents the cascade control design of a tubular chemical reactor. The procedure is based on a possibility of measuring the concentration of a main product of a reaction taking place in the reactor and measuring the temperature of the reactant at multiple points along the reactor. The control is performed in the external (primary) and inner (secondary) closed-loops where the concentration of a main product on the output of the reactor is the primary and the mean reactant temperature the secondary controlled variable. A common control input is the coolant flow rate.

The controller in the external control-loop is a discrete nonlinear P-controller derived on the basis of simulated or measured steady-state characteristics of the reactor. The controller in the inner control-loop is an adaptive LQ continuous-time controller. In its derivation, the recursive parameter estimation of an external delta model of the reactor, the polynomial approach and the LQ control theory are applied.

The presented method has been tested by computer simulation on the nonlinear model of the tubular chemical reactor with a consecutive exothermic reaction.

\section{References}

1. W.L. Luyben, Chemical reactor design and control (John Wiley \& Sons, Chichester, 2007)

2. D.E. Seborg, T.F. Edgar, D.A. Mellichamp, Process Dynamics and Control (John Wiley \& Sons, Chichester, 1989)

3. P. Dostál, V. Bobál, J. Vojtěšek, Proc. of 22nd European Conference on Modelling and Simulation, 487-492, Cyprus (2008).

4. J. F. Smuts, Process control for practitioners (OptiControls Inc., New York, 2011)

5. M. King, Process control: A practical approach (John Wiley \& Sons, Chichester, 2010)

6. R. Schmidt, Chemical process design and integration (John Wiley \& Sons, Chichester, 2005)

7. H. Garnier, L. Wang (eds.), Identification of continuous-time models from sampled data (SpringerVerlag, London, 2008)

8. S. Mukhopadhyay, A.G. Patra, G.P. Rao, Int. J. Contr. 55, (1992).

9. D.L. Stericker, N.K. Sinha, Control-theory and adv. Technol. 9, (1993)

10. V. Kučera, D. Henrion, Proc of IFAC Symposium Robust Control Design, Prague (2000)

11. J. Mikleš, M. Fikar, Process modellig, identification and control 2 (STU Press, Bratislava, 2004)

12. V. Bobál, J. Böhm, J. Fessl, J. Macháček, Digital selftuning controllers (Springer Verlag, Berlin, 2005) 